\title{
Shanxian granule ameliorates diethylnitrosamine-induced precancerous lesions in liver by regulating inflammatory mediators
}

\author{
Yan-fang PAN ${ }^{1}$, Xiao-tao JIA ${ }^{2 a}$, Xiao-ping YING ${ }^{1}$, Yan FANG ${ }^{1}$, Bo-bo MENG ${ }^{1}$ and Yang-QIAN ${ }^{1}$ \\ ${ }^{1}$ Department of Basic Medicine, Shaanxi University of Chinese Medicine, Xianyang, Shaanxi 712046, PR China \\ ${ }^{2}$ Department of Neurology, The Affiliated Xi'an Central Hospital of Xi'an Jiaotong University College of Medicine, Xi'an, Shaanxi \\ 710003, PR China
}

\begin{abstract}
To explore the effects of Shanxian Granules (SXG) on diethylnitrosamine (DEN) -induced precancerous lesions in rats and its possible molecular mechanism. A total of 50 male SD rats $(190 \pm 10 \mathrm{~g})$ were randomly divided into five groups. control group, DEN group, SXG low dose group, SXG medium dose group and SXG high dose group. The control group received saline injection. The precancerous liver cirrhosis group received $50 \mathrm{mg} / \mathrm{kg}$ DEN via intraperitoneal injection twice a week for 16 weeks. The indexes of liver function (ALT, AST and GGT) were measured by blood sampling. HE staining was used to observe pathological changes of liver tissues. The levels of IL- 6 and TNF $\alpha$ were measured by ELISA kits. Compared with the model group, the high $(P<0.01)$ and middle dose $(P<0.05)$ of SXG significantly reduced the damage of liver function and ameliorated the degree of liver disease, such as hepatic fibrosis. In addition, SXG significantly decreased the levels of IL-10 and TNF $\alpha$ in the liver of DEN-induced precancerous lesions. In conclusion, SXG can effectively and dose-dependently alleviate the morphological changes of rat liver precancerous lesions. and the underlying protective mechanism of SXG may be by inhibiting the release of inflammatory mediators, such as IL-6 and TNF- $\alpha$.
\end{abstract}

\section{Introduction}

Hepatocellular carcinoma (HCC) is one of the leading causes of cancer-related death around the world, and it is clinically characterized by a high incidence rate and poor prognosis [1]. Despite the progress made in numerous treatments, the survival rate of HCC patients remains low because HCC is not easily detected prior to the advanced stage [2]. Hepatitis, liver fibrosis-precancerous lesions and HCC is a malignant development process of chronic liver disease [3]. Therefore, if it can prevent or reverse the malignant changes of precancerous lesions of liver cancer, it may prevent the occurrence of liver cancer.

Precancerous lesions refer to benign liver lesions with potential for malignant transformation, including cirrhosis, regenerating nodules of hepatocytes, adenoma-like hyperplasia [4]. Some research believed that the formation of an inflammatory microenvironment provides microcirculation for liver precancerous lesions that is conducive to growth territory [5]. The chronic injury induces liver cell regeneration and repeated scar repair, which promotes liver fibrosis, while hepatic stellate cells (HSCs) play an important role in chronic liver inflammation, and activated HSCs produce extracellular matrix (ECM). IL-6 and TNF- $\alpha$ are the major inflammatory factors involved in this inflammatory response, and play a role in the process of precancerous lesions [6-9]. Therefore, we envisage whether it is possible to suppress or reverse its malignant transformation by regulating inflammatory mediators.

Shanxian Granules (SXG) is an anti-tumor Chinese traditional medicine developed by the oncology team of the department of integrated traditional Chinese people and western medicine of the affiliated hospital of shaanxi university of Chinese medicine. The results of previous studies have clarified the mechanism of SXG for the treatment of liver cancer [10], but whether it has the effect of inhibiting or reversing the malignant transformation of precancerous diseases has attracted our interest. Therefore, we used Intraperitoneal injection of DEN, representing a rodent model of HCC, to investigate whether SXG has protective effect against DEN-induced impairment of liver function and morphology, and whether SXG exerts these effects by reducing the inflammatory mediators in rats.

\section{Materials and Methods}

\subsection{Animals}

With approval of the Shaanxi Animal Research Ethics Committee, adult male Sprague-Dawley (SD) rats (180200g) supplied by the Research Animal Center of Xi'an Jiaotong University were housed in a room maintained at $23^{\circ} \mathrm{C}$ with a 12 -hour light-dark cycle. 


\subsection{Reagents and drugs}

Shanxian Granules (SXG) is composed of Chinese ginseng, Chinese turtle shell, turtle plate, zedoary turmeric, raw hawthorn, celestrus, salvia, barley, and porridge. (Production 20180204). Diethylnitrosamine (DEN): Provided by Shanghai Tixi Chemical Industrial Development Co., Ltd., the specification is $25 \mathrm{ml} /$ bottle (production batch number 20180309). HE Staining Kit (G1120, Solarbio). ELISA kits for IL-6 and TNF- $\alpha$ were from R\&D systems, Minneapo-lis, MN, USA.

\subsection{Animal groups and drug injections}

Sixty-eight rats were divided into five groups randomly, i.e. control group, DEN group, and SXG (2, 1 and $0.5 \mathrm{~g} / \mathrm{kg} /$ day) group plus DEN group. One week after the adaptive feeding of the rats, the rats were entered into the experiment. The prepared DEN was injected intraperitoneally ( $50 \mathrm{mg} / \mathrm{kg}$, twice a week) into the model group, SXG large, medium and small dose groups. Rats in the blank group were intraperitoneally injected with saline until the end of 16 weeks. Rats in the large, medium and small dose group of SXG were administrated with Chinese medicine $(2 \mathrm{~g} / \mathrm{kg}, 1 \mathrm{~g} / \mathrm{kg}, 0.5 \mathrm{~g} / \mathrm{kg})$ once a day; at the same time, the blank group and the model group were given the same dose of normal saline for intragastric administration, and the continuous administration was ended for 16 weeks.

\subsection{Material and index measurement}

After the last modeling and medication for 16 weeks, the rats were fasted for 24 hours. The rats were weighed and anesthetized with $10 \%$ chloral hydrate $(0.35 \mathrm{ml} / \mathrm{kg}$, intraperitoneal injection). The abdominal skin was disinfected with $75 \%$ alcohol and placed on a clean bench., laparotomy, blood from the inferior vena cava was sent to the department of biochemistry of the affiliated hospital of shaanxi university of traditional Chinese medicine to detect liver function-related indicators ALT, ALT and GGT. Then the level of IL- 6 and TNF- $\alpha$ in tissue lysates were checked by the commercial ELISA kits. The liver was removed from the abdominal cavity, and observe the liver color, texture, and whether there are nodules on the surface. If there are nodules, take two pieces of nodules surrounding liver, about $0.5 \mathrm{~cm} \times 0.5 \mathrm{~cm} \times 0.5 \mathrm{~cm}$. It was fixed in $10 \%$ formalin and paraffin-embedded.

\subsection{Histological Examination}

The liver tissues of each group were fixed, dehydrated, dipped in wax and embedded. It was sliced at a thickness of $3 \mu \mathrm{m}$, baked at $60^{\circ} \mathrm{C}$ for $1 \mathrm{~h}$, and then conventionally dewaxed to water. Take out the slide, soak the hematoxylin for 5-20min for staining. Then dehydration and transparency are performed, and the neutral gum is used for sealing; It is observed under a microscope after drying.

\section{Statistical Analysis}

All data were expressed as mean $\pm \mathrm{SD}$. One-way ANOVA followed by Tukey's post hoc test were performed in intergroup comparisons to determine the significant differences. All statistical analyses were performed by SPSS17.0. Statistical significance was accepted at $P<$ 0.05 .

Table 1 The level of ALT, AST and GGT in blood of rats (Mean \pm SEM, $n=10$ )

\begin{tabular}{cccc}
\hline Groups & ALT(U/L) & AST(U/L) & GGT(U/L) \\
\hline Control & $37.25 \pm 9.39$ & $102.36 \pm 6.87$ & $1.76 \pm 0.17$ \\
DEN & $328.27 \pm 12.35^{*}$ & $288.90 \pm 8.23^{*}$ & $154.80 \pm 8.12^{*}$ \\
SXG $(0.5 \mathrm{~g} / \mathrm{kg} / \mathrm{d})$ & $282.26 \pm 16.96$ & $242.18 \pm 8.02$ & $98.09 \pm 5.42$ \\
SXG $(1 \mathrm{~g} / \mathrm{kg} / \mathrm{d})$ & $187.75 \pm 9.82^{\#}$ & $177.31 \pm 7.71^{\#}$ & $67.46 \pm 5.81^{\#}$ \\
SXG $(2 \mathrm{~g} / \mathrm{kg} / \mathrm{d})$ & $139.69 \pm 11.31^{\# \#}$ & $156.42 \pm 4.83^{\# \#}$ & $45.17 \pm 5.87^{\# \#}$ \\
\hline$* P<0.05$ compared with the control group $^{\#} P<0.05$ and $P<0.01$ compared with the DEN group, respectively
\end{tabular}

${ }^{*} P<0.05$ compared with the control group, ${ }^{\#} P<0.05$ and ${ }^{\# \#} P<0.01$ compared with the DEN group, respectively

\section{Results}

\subsection{SXG effectively prevented the DEN-induced decrease of ALT, AST and GGT in serum of rat}

During the development of precancerous lesions of the liver, hepatocytes are gradually damaged and liver function is gradually reduced. Therefore, at the end of this experiment, we collected venous blood from animals in each group and measured changes in liver function related indicators ALT,AST,GGT.AS shown in Table 1,compared with the blank group, the levels of ALT,AST and GGT in the model group were significantly higher than those in the blank group $(P<0.05)$. Compared with the model group, the ALT, AST, and GGT in the low-dose SXG group were all reduced. But the difference was not statistically significant $(P>0.05)$. However, ALT, AST, and GGT in the SXG large and medium dose groups were significantly reduced, and the differences were statistically significant $(P<0.05)$. These results indicated that the treatment of SXG ( 1 and $2 \mathrm{~g} / \mathrm{kg} /$ day $)$ can significantly reduce the decline of liver function in DENinduced hepatocellular carcinoma model rats. 


\subsection{SXG treatment prevented against DEN- induced precancerous lesions impairment in a dose-dependent manner}

In order to further observe the changes in the structure of the liver, we made paraffin sections of the liver tissue of each treatment group and performed HE staining. As shown in Fig1. The normal rat livers showed typical lobular architecture and uniform distribution of liver cells visualized with HE staining., hepatocytes in the blank group had normal morphology. The veins are arranged radially, without obvious edema, steatosis, necrosis and fibrous hyperplasia. However, in model group, hepatocytes edema and deformation, intracellular fat deposition, local parenchymal cells necrosis, dilated bile ducts, cholestasis, and large bile ducts hyperplasia; hepatic parenchymal nucleus swells with nuclear division polymorphism, accompanied by dysplasia and nodule formation, disordered arrangement, hyperplasia collagen fibers are destroyed to form pseudolobules, and lymphocytes move to the liver intrasinus and manifold areas migrate. Compared with the blank group, the lesion score of liver the model group were significantly improved $(P<0.05)$. Rats SXG $(0.5 \mathrm{~g} / \mathrm{kg} /$ day $)$ have disordered liver cell arrangement, showing a large number

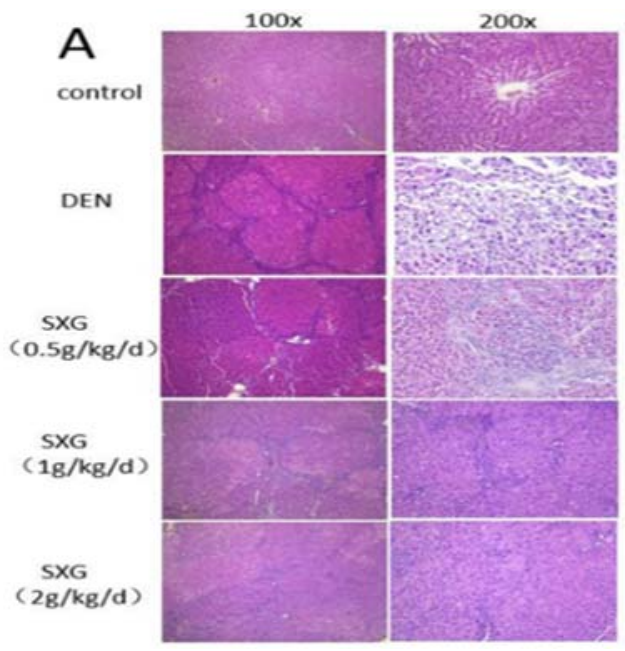

of cellular edema, lipid change, and necrosis. Pseudolobularity is generated in liver tissue, but fewer than the model group $(P>0.05)$. Rats of SXG $(1 \mathrm{~g} / \mathrm{kg} /$ day $)$ have less hepatocytes arranged than the model group. No significant pseudolobules were found in the tissues, and a large number of fibroproliferation, edema, lipid change, and necrosis were observed, but it was reduced compared with the model group and the SXG $(0.5 \mathrm{~g} / \mathrm{kg} /$ day $)$ group; The liver cells of the $\mathrm{SXG}(2 \mathrm{~g} / \mathrm{kg} /$ day $)$ group were basically normal, and there were a small amount of fibroproliferation and cells edema, steatosis, and necrosis were reduced to a lesser extent than in $\operatorname{SXG}(0.5 \mathrm{~g} / \mathrm{kg} /$ day $)$ group and DEN group $((\mathrm{p}<0.05)$. These results indicate that the treatment of SXG (1 and $2 \mathrm{~g} / \mathrm{kg} /$ day) can significantly prevented against DEN-induced precancerous lesions impairment

\subsection{SXG markedly suppressed inflammatory cytokines accumulation induced by DEN in rat}

Inflammatory mediators is strongly correlated with hepatocyte dysfunctions in the pathophysiology of precancerous lesions of HCC. To further elucidate the probable biochemical mechanism of the protective effect

Figure 1. The pathological images of normal and cirrhotic precancerous lesions of rat livers

A: Hematoxylin-eosin staining, magnification $\times 100$ and $\times 200$ respectively.

B: The histograms summarize the effects of the coapplication of different dosages of SCG and DEN on the precancerous lesions.

The data were represented as mean \pm S.E.M. $(\mathrm{n}=10) .{ }^{*}$ $P<0.05$ v.s. control group. ${ }^{\#} P<0.05$ and ${ }^{\#} P<0.01$ v.s DEN alone group of SCG in DEN-mediated impairment in precancerous lesions, we subsequently tested whether SCG influenced the release of inflammatory mediators. In this study, the levels of two proinflammatory cytokines (IL-6 and TNF- $\alpha$ ) in liver were measured. ELISA results showed that IL- 6 and TNF- $\alpha$ levels were significantly increased compared to control group as shown in Fig.2 Meanwhile, the treatment of SXG ( 1 and $2 \mathrm{~g} / \mathrm{kg} /$ day) significantly attenuated the DEN-induced up-regulation of those two proinflammatory cytokines in the rats. The results suggest that SCG prevented the pro-inflammatory cytokines accumulation induced by DEN in rat liver. 

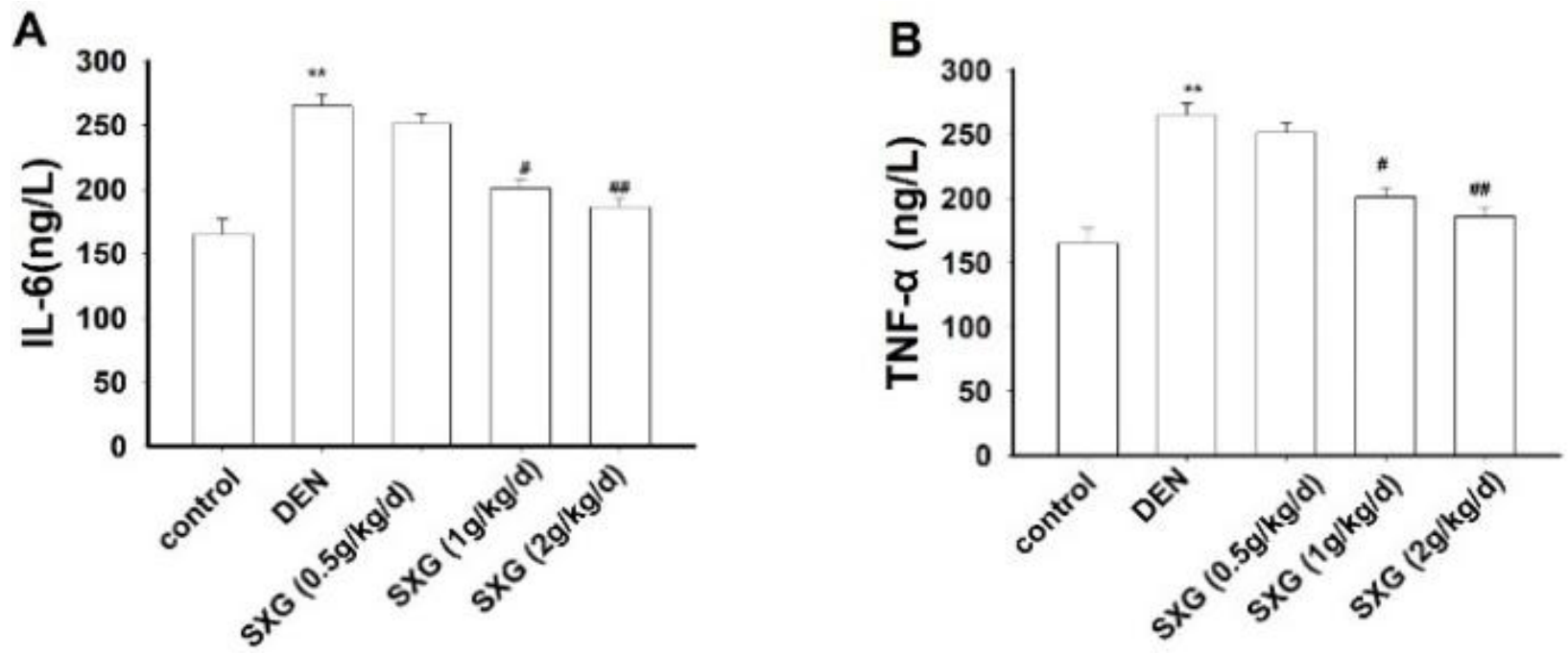

Figure 2.SCG prevented the increase of IL-6 and TNF- $\alpha$ induced by DEN

ELISA for IL-6(A) and TNF- $\alpha$ (B) were performed in the rats with various treatments. Values are expressed as mean \pm SEM $(\mathrm{n}=6)$. ** $P<0.01$ v.s control group; ${ }^{\#} P<$ 0.05 and $^{\# \#} P<0.01$ v.s DEN group respectively.

\section{Discussion}

HCC is among the leading global healthcare issues associated with high morbidity and mortality. Early detection of pathological changes before the precancerous lesions occur is critical for the treatment and prognosis of the disease. DEN-induced HCC in rat is an experimental model that mimics the pathological process of human HCC [11-13]. In the present study, we made a rat model of liver cirrhosis with DEN-induced precancerous lesions, and observed the effects of Shanxian granules on precancerous lesions. It was found that the treatment of SXG (1 and $2 \mathrm{~g} / \mathrm{kg} /$ day) could significantly improve liver function and relieve liver lesions in rats with precancerous lesions. ALT and AST are the most commonly used indicators in the eva- luation of clinical liver function [14]. ALT is mostly found in liver cells in the cytoplasm, AST is mainly found in the mitochondria of liver cells. When liver cells are damaged, both are released intracellularly radiation, the amount of its serum can reflect the degree of liver damage. GGT is a specific biochemical indicators in liver cancer tissue. Some studies have shown that the positive rate of GGT for liver cirrhosis is $75 \%$ and has a higher positive diagnosis rate in liver cancer, reaching $97.6 \%$ [15]. The results in this study showed that compared with the blank group, liver function index levels of rats in DEN group increased significantly, indicating the success of experimental modeling However, Administration of SCG $(25,50 \mathrm{mg} / \mathrm{kg})$ significantly and dose-dependently suppressed reduce the serum ALT, AST, GGT. These results that SCG has a significant effect on liver function improvement effect.

Chronic inflammatory response induces liver cancer formation, and various inflammatory factors are the main effectors that mediate this process. TNF- $\alpha$ is a proinflammatory factor, and was secreted by activated monocytes / macrophages [16]. When TNF- $\alpha$ is excessively released, it can cause pathological damage. In the precancerous process of liver fibrosis-cirrhosis-liver process of liver fibrosis-cirrhosis-liver cancer, TNF- $\alpha$ mainly plays a role in triggering chronic inflammatory reactions to change the liver cell microenvironment, and long-term stimulation of molecular substances that cause liver cells to undergo gene mutation, dysplasia, and promote tumor formation, leading to tumor formation, invasion and metastasis [17]. IL-6, as one of the main factors that mediate inflammatory response, plays an important role in the formation of liver injury, liver fibrosis, cirrhosis and even liver cancer. Recent studies have found that IL-6-induced liver inflammatory damage by oxidative damage [18]. Another study found that IL-6 levels in liver tumor cells are abnormally increased, which is related to tumor malignancy and infiltration [8]. The results in this study showed that TNF- $\alpha$ and IL-6 and levels in DEN group were significantly increased compared to control group. However, Administration of SCG $(1,2 \mathrm{~g} / \mathrm{kg})$ significantly and dose-dependently suppressed the inflammatory responses in DEN-treated rats, suggesting the anti-neuroinflammation role of SCG in precancerous lesions.

In summary, our results indicated that SXG dosedependently ameliorate DEN-induced liver function and morphology impairments in rats. The reduction of proinflammatory cytokines release are accountable for the protective effects of SXG against DEN induced injury in the liver. It is necessary to further clarify the detailed mechanism of SXG in the treatment of precancerous lesions. Therefore, SXG may be an effective therapeutic agent in preventing the development of precancerous lesions of the liver.

\section{Acknowledgements}

This research was supported by the National Science Foundation of PR China (81703842), traditional Chinese medicine scientific research projects of Shaanxi province of PR China (JCMS032) and Innovation and Entrepreneurship Training Program of College Students of Shaanxi University of Chinese Medicine 


\section{(S201910716059).}

\section{References}

1. G.Rong, H.Wang, C.L.Bowlus, Incidence and risk factors for hepatocellular carcinoma in primary biliary cirrhosis.[J]. Clin. Rev. Allergy Immunol, 48,1-10 (2015)

2. L.D. Tommaso, A. Sangiovanni, M. Borzio, Y.N. Park, F. Farinati, M. Roncalli, Hepatocellular carcinoma pathogenesis: from genes to environment[J]. Nat. Rev. Cancer, 6:674-687(2006)

3. A. Sangiovanni, M. Borzio, Advanced precancerous lesions in the liver [J]. Best. Pract. Act. Res. Cl. Ga, 27, 269-84 (2013)

4. G. Li, L.X. Wang., R. Pin, Dysplastic Nodules with Glypican-3 Positive Immunostaining: A Risk for Early Hepatocellular Carcinoma[J]. PLoS One, 9,1 (2014)

5. Z.J. Lawless, W. Matthew, Stop feeding cancer: Proinflammatory role of visceral adiposity in liver cancer[J]. Cytokine, 64, 626-637(2013).

6. V. Carloni, T.V. Luong, K. Rombouts. Hepatic stellate cells and ECM in HCC: more complicated than ever[J]. Liver international: official journal of the International Association for the Study of the Liver, 34,6(2014)

7. K. Y. Murata, H. Ishiguchi, R. Ando, Chronic inflammatory demyelinating polyneuropathy associated with primary biliary cirrhosis[J]. J. Clin. Neurosci., 20,1799-1801(2013)

8. E. J .Park, J .H.Lee, G .Y .Yu ,. Dietary and Genetic Obesity Promote Liver Inflammation and Tumorigenesis by Enhancing IL-6 and TNF Expression[J]. Cell, 140,2. (2010)

9. R. J. Stefa, IL-6 Trans-Signaling via the Soluble IL-6 Receptor: Importance for the Pro-Inflammatory
Activities of IL-6[J]. Int. J. Biol. Sci., 8,9(2012)

10. Y. Fang, X.P. Ying, X,P Wang, Effect of Shanxian Granule-containing serum on the expression of caspase- 3 and heat shock protein 70 in S180 cells [J]. Chinese Traditional Patent Medicine, 9,178-180(2014)

11. H. Koen, T.D. Pugh, S. Goldfarb, Centrilobular distribution of diethylnitrosamine-induced hepatocellular foci in the mouse[J]. LAB INVEST, 49,78-81(1983)

12. P. Newell, A. Villanueva, S. L. Friedman, Experimental models of hepatocellular carcinoma[J]. J. Hepatol., 48,5 (2008)

13. H. J. Hacker, H. Mtiro, P. Bannasch,. Histochemical profile of mouse hepatocellular adenomas and carcinomas induced by a single dose of diethylnitrosamine.[J]. Cancer Res., 51,1952-1958 (1991)

14. E .Giannini, F. Botta , A. Fasoli, P.. Ceppa, D .Risso, Progressive Liver Functional Impairment Is Associated with an Increase in AST/ALT Ratio[J]. Dig. Dis. Sci. 44,1249-1253 (1999)

15. C. Cray, D. Gautier, D.J. Harris,. Changes in Clinical Enzyme Activity and Bile Acid Levels in Psittacine Birds With Altered Liver Function and Disease[J]. J. Avian Med. Surg., 22,17-24 (2008)

16. I. Nel, O. Lucar, C. Petitdemange,. Accumulation of Intrahepatic TNF- $\alpha$-Producing NKp44+ NK Cells Correlates With Liver Fibrosis and Viral Load in Chronic HCV Infection[J]. Medicine, 95,19 (2016)

17. A. H. Harken. TLR4 mediates LPS-induced HO-1 expression in mouse liver: Role of TNF- $\alpha$ and IL$1 \beta[\mathrm{J}]$. World J. Gastroenterol., 9,1799-1803. (2003)

18. L. Tacchini, G.Cairo, D.P.C. , M. Massip, R.C.Joan, C. Peralta, Up regulation of IL-6 by ischemic preconditioning in normal and fatty rat livers: Association with reduction of oxidative stress[J]. Free Radic. Res., 40,1206-1217(2006) 